\title{
The Use of Fluorescence In Situ Hybridization in Bacterial Detection in Mycorrhizal Networks
}

\author{
Will Chrisler ${ }^{1}$, Gayla Orr ${ }^{2}$, Alice Dohnalkova ${ }^{2}$, Ljiljana Paša-Tolić ${ }^{2}$, Gary Stacey ${ }^{3}$, Chanlan Chun $^{4}$ \\ Michael Sadowsky ${ }^{4}$ \\ ${ }^{1}$ Health Impacts \& Exposure Sciences Division, Pacific Northwest National Laboratory, Richland, WA \\ ${ }^{2}$ Environmental Molecular Sciences Laboratory, Pacific Northwest National Laboratory, Richland, WA \\ ${ }^{3}$ Division of Plant Sciences, National Center for Soybean Biotechnology, Christopher S. Bond Life \\ Sciences Center, University of Missouri, Columbia, Missouri \\ ${ }^{4}$ BioTechnology Institute, University of Minnesota
}

Rhizosphere, the belowground biogeochemical hotspot of plant roots intimately associated with soil bacteria and plant fungi (mycorrhizae), is a highly interactive microcosm where fundamental processes such as carbon fixation, nutrient acquisition and nitrogen cycling occur. The detailed exploration of these micro-scale rhizosphere processes is essential to our better understanding of large-scale processes in soils, and determination what drives source-sink relationships that control the cycling of plant nutrients belowground. To study the nutrient partitioning into roots and their exchange with the soil microbial community, a multidisciplinary, multi-institutional research project was designed to address the complexity of the plant-soil interface, using the means of genomics, and correlated analytical tools including methods of mass spectroscopy (analyzing proteomic, metabolomic and transcriptomic components) and high resolution microscopy.

Arabidopsis thaliana wild-type and SEX (=starch excess) mutants were grown hydroponically or in artificial potting mix, and rhizosphere population arising from inoculation with soil extract was established and analyzed by a variety of tools to view the live interactions of plant roots with the soil microbial population. The SEX mutants lack the ability to allocate starch (stored carbon) from their leaves to the rest of the plant during the dark period. Research has shown that the growth differs significantly in SEX mutants from the wild-type in carbon allocation to the roots [1]. Our focus will be to develop a method to examine how the mutated genome affects the spatial and temporal differences in colonization between the bacteria and roots of $A$. thaliana.

The challenge to this complex system is to differentiate the bacteria associated with the plant root fro the root itself. Many dyes are not specific to a single organism and will not stain one community member independently. Using a combination of species specific fluorescent stains, non-specific fluorescent stains, and FISH probes, the spatial distribution of the bacteria-root environment can be investigated. Propidium iodide allows the detection of DNA in both bacteria and the $A$. thaliana roots. Using Calcofluor White, the cellulose in A. thaliana root can be specifically stained to determine the root boundary. The probe EUB 338, specific for the domain Bacteria, was used to identify bacteria. $A$. thaliana grown with/without bacterial inoculant derived from soil extract are harvested prior to the beginning of light and dark cycles. Roots are washed in PBS and then fixed in 4\% paraformaldehyde for 30 minutes at room temperature. The samples were then washed in PBS and kept in $4{ }^{\circ} \mathrm{C}$. Segments of the roots were dissected from A. thaliana wild-type and Sex-like mutants, and processed for Fluorescence in situ hybridization (FISH) with rRNA-targeted probes following a slightly modified protocol [2]. The FISH labeled samples are then imaged by confocal laser scanning microscopy. 
Additional morphological determination of the microbial community was done by scanning electron microscopy (SEM).

[1] Weber A. et al. The Plant Cell (13), 1907-1918. ( 2001)

[2] Hugenholtz P. et al. Methods in Molecular Biology (179), 2002, pp 29-42

[3] This research was performed at the W. R. Wiley Environmental Molecular Sciences Laboratory, a national scientific user facility sponsored by the U.S. DOE, located at PNNL.
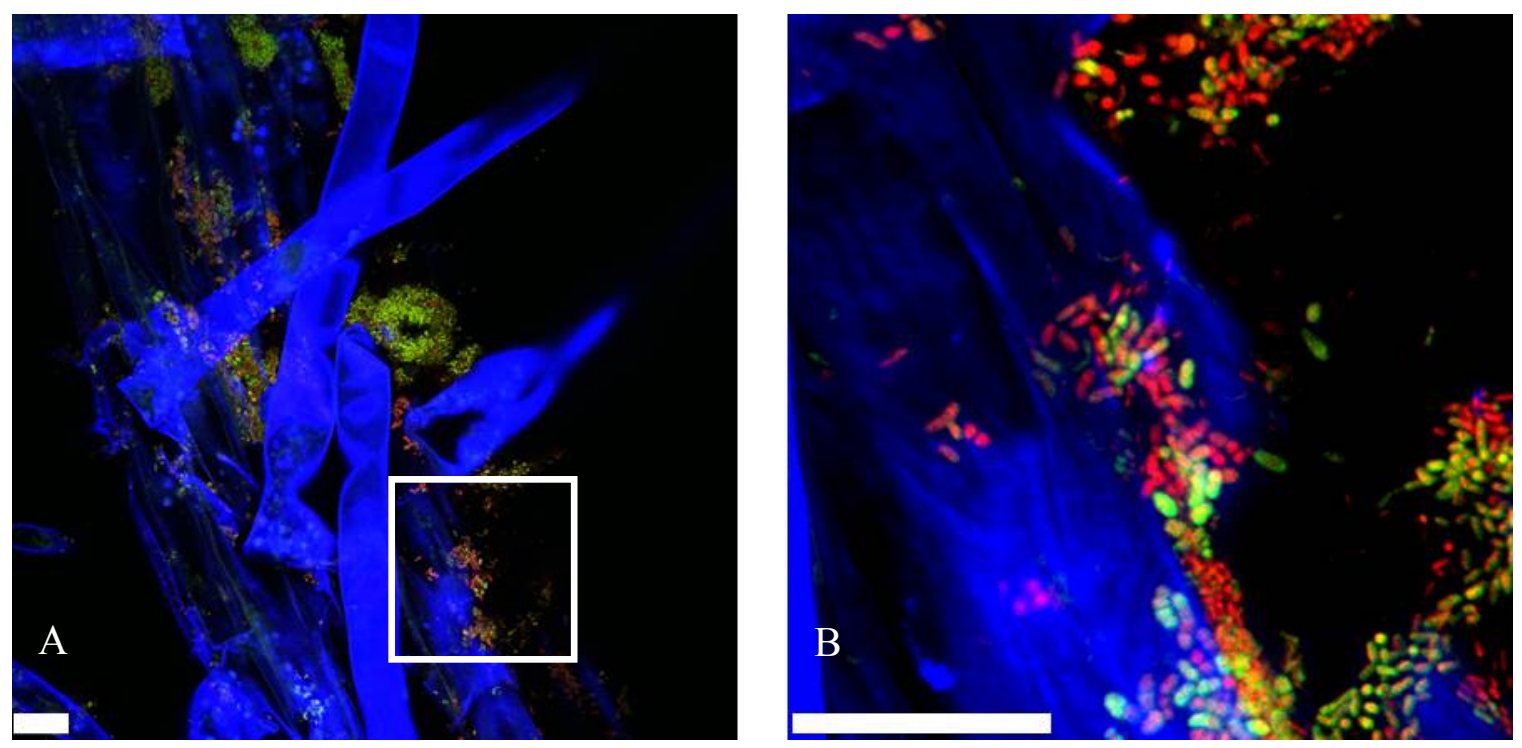

Figure 1. (A) Confocal laser scanning microscopy (CLSM) micrographs showing the bacterial colonization of $A$. thaliana roots stained by fluorescence in situ hybridization (FISH). (green, EUB-338 AlexaFluor488; blue, root fluorescence; red, DNA). (B) Increased magnification of region indicated by box in (A). Scale Bar 20um 